\title{
ON BOUNDED SOLUTIONS TO CONVOLUTION EQUATIONS
}

\author{
ENRICO PRIOLA AND JERZY ZABCZYK
}

(Communicated by Jonathan M. Borwein)

\begin{abstract}
Periodicity of bounded solutions for convolution equations on a separable abelian metric group $G$ is established, and related Liouville type theorems are obtained. A non-constant Borel and bounded harmonic function is constructed for an arbitrary convolution semigroup on any infinite-dimensional separable Hilbert space, generalizing a classical result by Goodman (1973).
\end{abstract}

\section{INTRODUCTION}

Let $\mu$ be a probability measure defined on the $\sigma$-algebra $\mathcal{B}(G)$ of Borel subsets of a separable abelian metric group $G$, with the group operation "+". This group might be non-locally compact. This paper is concerned with convolution equations of the type

$$
f * \mu(x):=\int_{G} f(x-y) \mu(d y)=f(x), \quad x \in G,
$$

where $f: G \rightarrow \mathbb{R}$ is a Borel and bounded function, written $f * \mu=f$ for short.

Our aim is to investigate bounded Borel solutions $f$ to (1.1). These functions are also called bounded $\mu$-harmonic functions; see [6]. Special attention will be paid to the case when $G$ is a real separable Hilbert space.

Convolution equations arise naturally in several areas of pure and applied mathematics such as harmonic analysis (see [6], 4] and [15]), the theory of Markovian semigroups (see [9], 1] and [11]), and the renewal theory (see [10]).

Define the shift $\mu_{h}$ of the measure $\mu$ by the element $h \in G$, through the formula

$$
\mu_{h}(A)=\mu(A-h), \quad A \in \mathcal{B}(G) .
$$

Consider the set $M_{\mu} \subset G$ of all elements $h \in G$ such that measures $\mu_{h}$ are absolutely continuous with respect to $\mu$. The set $M_{\mu}$ has been introduced in 12 and investigated, for instance, in [24, 23] and [2]. Our main result (see Theorem 2.5) shows that each Borel and bounded solution $f$ to (1.1) is periodic with periods in $M_{\mu}$ i.e.,

$$
f(x+h)=f(x), \quad x \in G, h \in M_{\mu} .
$$

Received by the editors May 25, 2005.

2000 Mathematics Subject Classification. Primary 43A55, 68B15, 47D07, 31C05.

Key words and phrases. Convolution equations on groups, bounded harmonic functions, Lévy processes.

The first author was partially supported by Italian National Project MURST "Equazioni di Kolmogorov" and by Contract No ICA1-CT-2000-70024 between European Community and the Stefan Banach International Mathematical Center in Warsaw. 
The theorem holds in the more general setting of measurable abelian groups; see Remark 2.11, Moreover the set $M_{\mu}$ can be replaced by a larger set $E_{\mu}$; see (2.1).

The result seems particularly useful in infinite dimensions, when $G$ is a separable Hilbert space $H$ and there is no reference to any Haar measure. In Section 3.1 we consider an application to $\alpha$-stable measures $\mu$ on $H, \alpha \in(0,2]$.

A related equation,

$$
\nu * \mu=\nu
$$

on a locally compact group $G$, with unknown non-negative measure $\nu$, was the subject of a classical paper [3] by Choquet and Deny. Under suitable assumptions on $\nu$ (see Section 2.3), they proved that $\nu$ is periodic with periods in the subgroup generated by the support $S_{\mu}$ of $\mu$ (i.e., $S_{\mu}$ is the smallest closed set of $G$ on which $\mu$ is concentrated). Within the class of locally compact groups, this result and related Liouville theorems have been extended in several directions; see [6, [15], 4], 21] and the references therein.

Even when $G$ is locally compact, our result does not follow from [3]; see Remark 2.8. Moreover (1.2) does not hold if $M_{\mu}$ is replaced by $S_{\mu}$. On the other hand, as a consequence of our main result, in Proposition 2.12 we obtain a version of the Choquet-Deny theorem concerning (1.3), which holds on metric groups (replacing the subgroup generated by $S_{\mu}$ with a smaller subgroup which contains $M_{\mu}$ ). We also show in Theorem 2.9 that uniformly continuous and bounded solutions to (1.1) are periodic with periods in $S_{\mu}$ (see also Corollary 3.3.2 in [20]).

Equation (1.1) and related Liouville type theorems on a separable Hilbert space $H$ are considered in Section 3. Let $\left(\mu_{t}\right)_{t \geq 0}$ be a convolution semigroup of probability measures on $H$. A function $f \in B_{b}(H)$ such that, for any $t \geq 0$,

$$
f * \mu_{t}(x)=f(x), \quad x \in H,
$$

is called a bounded harmonic function with respect to $\left(\mu_{t}\right)$. In Theorem 3.3 we prove that if the space $H$ is infinite dimensional, then one can always construct a discontinuous non-constant solution $f$ to (1.4). This result generalises a well-known example of Goodman [14, concerning the case when $\left(\mu_{t}\right)$ are Gaussian measures. The Goodman result shows clearly that (1.2) cannot hold if $M_{\mu}$ is replaced by $S_{\mu}$. This was one motivation for us to investigate (1.1).

Theorem 3.3 also implies that Markovian convolution semigroups associated to $\left(\mu_{t}\right)$ are never strong Feller in infinite dimensions; cf. [7.

\section{Convolution equations on metric groups}

Let $G$ be a separable abelian metric group with group operation indicated by + ; see [16] and 23]. For $A, B \subset G$, we set

$$
A+B=\{x+y, \quad x \in A, y \in B\}, \quad-A=\{-x, x \in A\} .
$$

Moreover $\operatorname{Gr}(A)$ denotes the smallest subgroup containing $A$, and $\bar{A}$ denotes the closure of $A$. The indicator function of a set $F \subset G$ will be indicated by $1_{F}$.

When $G$ is complete, we call it an abelian Polish group. When $G$ is a real separable Hilbert space, we denote it by $H$. The inner product of $H$ is then $\langle\cdot, \cdot\rangle$ and its norm is $|\cdot|$.

The probability measures on $G$ we consider will always be Borel probability measures on the $\sigma$-algebra $\mathcal{B}(G)$. Let $\sigma$ be a probability measure on $G$. The 
support of $\sigma$ is denoted by $S_{\sigma}$; it is the smallest closed set in $G$ which has measure 1 with respect to $\sigma$.

With $\tilde{\sigma}$ we indicate its reflection measure with respect to 0 (see [22, Chapter $1]$ ), i.e. $\tilde{\sigma}(A)=\sigma(-A)$ for any $A \in \mathcal{B}(G)$. The probability measure $\sigma$ is called symmetric if $\sigma=\tilde{\sigma}$, i.e. $\sigma(A)=\sigma(-A), A \in \mathcal{B}(G)$.

For $\mu$ and $\nu$ probability measures on $G$, the convolution measure $\mu * \nu$ is defined by $\mu * \nu(A):=\int_{G} \mu(A-x) \nu(d x), A \in \mathcal{B}(G)$; see for instance [16] or [23]. Note that the operation $*$ is commutative and associative.

We write $\mu^{n}=\mu * \cdots * \mu$ (n-times), $n \in \mathbb{N}$ ( $\mathbb{N}$ denotes the set of all positive integers). We also set $\mu^{0}=\delta_{0}$, where $\delta_{0}$ is the Dirac measure concentrated in 0 .

By $B_{b}(G)$ we denote the Banach space of all real, Borel and bounded functions $f: G \rightarrow \mathbb{R}$, endowed with the supremum norm $\|\cdot\|_{\infty}$. If $g \in B_{b}(G)$, we set

$$
g * \mu(x)=\int_{G} g(x-y) \mu(d y), \quad x \in G .
$$

2.1. Admissible shifts. Let $T_{a}, a \in G$, be the translation operator, i.e. $T_{a}(x)=$ $x+a, x \in G$, and denote by $T_{a} \circ \mu$ the image of a probability measure $\mu$ under $T_{a}$, i.e. $\left(T_{a} \circ \mu\right)(A)=\mu(A-a), A \in \mathcal{B}(G)$. We also set $\left(T_{a} \circ \mu\right)=\mu_{a}$.

According to [12, page 449], $a \in G$ is called an admissible shift for $\mu$ if $T_{a} \circ \mu$ is absolutely continuous with respect to $\mu$. Let us denote by $M_{\mu}$ the set of all admissible shifts. Note that $0 \in M_{\mu}$. Moreover it is known that $M_{\mu}$ is always a semigroup of $G$; see [12, page 450]. Since $M_{\tilde{\mu}}=-M_{\mu}$, where $\tilde{\mu}$ is the reflection measure of $\mu$ (see [24]), we have that $M_{\mu}$ is a subgroup of $G$ if $\mu$ is symmetric.

If $G$ is locally compact and $\mu$ is equivalent to the Haar measure of $G$, then it is easy to show that $M_{\mu}=G$.

We introduce the set

$$
E_{\mu}=\bigcup_{n \geq 0} M_{\mu^{n}}, \quad \mu^{n}=\mu * \cdots * \mu(n \text {-times }) n \in \mathbb{N} .
$$

We will need the following elementary result.

Proposition 2.1. Let $\mu$ and $\nu$ be probability measures on $G$. Then

$$
M_{\mu}+M_{\nu} \subset M_{\mu * \nu}
$$

Moreover $E_{\mu}$ is a semigroup of $G$.

Proof. Take any $A \in \mathcal{B}(G)$ such that $\mu * \nu(A)=0$ and $a \in M_{\mu}, b \in M_{\nu}$. It is enough to show that $(\mu * \nu)_{a+b}(A)=0$. We have

$$
(\mu * \nu)_{a+b}(A)=\int_{G} \int_{G} 1_{A}(x+y) p(x) q(y) \mu(d x) \nu(d y)
$$

where $p$ and $q$ are the densities of $\mu_{a}$ and $\nu_{b}$, respectively. For any $N \in \mathbb{N}$, set $p_{N}=N \wedge p$ and $q_{N}=N \wedge q$. Then

$$
\int_{G} \int_{G} 1_{A}(x+y) p_{N}(x) q_{N}(y) \mu(d x) \nu(d y) \leq N^{2} \int_{G} \int_{G} 1_{A}(x+y) \mu(d x) \nu(d y)=0 .
$$

Taking $N \rightarrow \infty$, we get the first assertion. This implies that $E_{\mu}$ is an increasing union of semigroups. The second assertion follows easily.

The next example shows that the inclusion in (2.2) can be strict. 
Example 2.2. There exists a probability measure $\mu$ on $\mathbb{R}^{n}$ such that $M_{\mu}=0$ and $M_{\mu^{2}}=\mathbb{R}^{n}=E_{\mu}$.

Define $\mu=\sum_{k \geq 1} p_{k} \nu_{k}$, where $\nu_{k}$ are uniform distributions on the spheres centered in 0 , of radiuses $k \in \mathbb{N}$, and $\sum_{k \geq 1} p_{k}=1, p_{k}>0, k \in \mathbb{N}$. Since the measures $\nu_{k} * \nu_{l}$ are absolutely continuous with respect to Lebesgue measure (see [10]) and the density of $\nu_{k} * \nu_{k}$ is positive on the ball $B(0,2 k)$, we have $\mu * \mu=\sum_{k, l=1}^{\infty} p_{k} p_{l} \nu_{k} * \nu_{l}$, and $\mu * \mu$ has a positive density on $\mathbb{R}^{n}$. The assertion follows.

We now compare the set $E_{\mu}$ with the subgroup generated by the support $S_{\mu}$ of $\mu$.

Proposition 2.3. Let $\mu$ be a probability measure on $G$. Then $E_{\mu} \subset \operatorname{Gr}\left(S_{\mu}\right)$.

Proof. Fix any $h \in M_{\mu}$. It is straightforward to check that $S_{\mu}+h \subset S_{\mu}$.

Now take $x \in S_{\mu}, x \neq 0$ (if $S_{\mu}=\{0\}$, then $\mu$ is the Dirac measure concentrated in 0 and $M_{\mu}=\{0\}$ as well). We know that $x+h \in S_{\mu} \subset \operatorname{Gr}\left(S_{\mu}\right)$. Since also $-x \in$ $\operatorname{Gr}\left(S_{\mu}\right)$, it follows that $h \in \operatorname{Gr}\left(S_{\mu}\right)$. We have proved that $M_{\mu} \subset \operatorname{Gr}\left(S_{\mu}\right)$.

For any $n \in \mathbb{N}$, one has $M_{\mu^{n}} \subset \operatorname{Gr}\left(S_{\mu^{n}}\right)=\operatorname{Gr}\left(S_{\mu}\right)$. Hence the assertion holds.

In general the sets $M_{\mu}$ and $S_{\mu}$ are different.

Example 2.4. There exists a probability measure $\mu$ on $\mathbb{R}^{n}$ such that $M_{\mu}$ and $S_{\mu}$ are disjoint sets.

Take $x_{0} \in \mathbb{R}^{n}, x_{0} \neq 0$, and $u \in \mathbb{R}^{n}$ such that $|u|=1$. Consider the line $L=\left\{x \in \mathbb{R}^{n}: x=\lambda u+x_{0}\right.$, for $\left.\lambda \in \mathbb{R}\right\}$.

Let $\mu$ be a probability measure on $\mathbb{R}^{n}$, concentrated on $L$, having a positive density with respect to the one-dimensional Lebesgue measure. We have that $M_{\mu}=$ $\{\lambda u\}_{\lambda \in \mathbb{R}}$ and $S_{\mu}=L$.

\subsection{Characterization theorems.}

Theorem 2.5. Let $G$ be a separable abelian metric group. Let $\mu$ be a probability measure on $G$ and let $E_{\mu} \subset G$ be defined in (2.1). Let $f \in B_{b}(G)$ be a $\mu$-harmonic function. Then one has

$$
f(x+a)=f(x), \quad x \in G, \quad a \in G r\left(E_{\mu}\right) .
$$

If, in addition, $f$ is continuous on $G$ and $G r\left(E_{\mu}\right)$ is dense in $G$, then $f$ is constant.

The proof uses the following result; see [8, Theorem 9, page 292].

Theorem 2.6. Let $(\Omega, \mathcal{F}, \nu)$ be a measure space (with $\nu$ a positive measure). Let $K$ be a bounded subset of $L^{1}(\Omega, \nu)$. Assume that, for each decreasing sequence $\left(E_{n}\right) \subset \mathcal{F}$ with empty intersection, the limit $\lim _{n \rightarrow \infty} \int_{E_{n}} f(s) \nu(d s)=0$ is uniform with respect to $f \in K$. Then, for any sequence $\left(f_{n}\right) \subset K$, there exists a subsequence $\left(f_{n_{k}}\right)$ which converges weakly in $L^{1}(\Omega, \nu)$.

Proof of Theorem 2.5. We first define, similar to [3], suitable auxiliary functions and then obtain the required characterization arguing by contradiction. Both steps are accomplished differently from 3. In particular, instead of the Ascoli-Arzela theorem, we use arguments based on $L^{1}$-weak compactness.

Let us introduce $\tilde{f}, \tilde{f}(x)=f(-x), x \in G$. The equation $f * \mu=f$ is equivalent to

$$
\tilde{f} * \tilde{\mu}=\tilde{f},
$$


where $\tilde{\mu}$ is the reflection measure of $\mu$. Fix $a \in M_{\mu}$ and introduce the function

$$
g(x)=\tilde{f}(x)-\tilde{f}(x+a) .
$$

It is clear that $g \in B_{b}(G)$ and $g * \tilde{\mu}=g$ on $G$. Let

$$
2 c=\sup _{x \in G} g(x)
$$

and $\left(x_{n}\right) \subset G$ such that $g\left(x_{n}\right) \rightarrow 2 c$ as $n \rightarrow \infty$. Consider the functions $g_{n}: G \rightarrow \mathbb{R}$,

$$
g_{n}(x)=g\left(x+x_{n}\right), \quad x \in G .
$$

Each $g_{n} \in B_{b}(G)$ and solves the convolution equation (2.3). Now we set $L^{1}=$ $L^{1}(G, \mu)$ and use $L^{1}$-weak convergence $\left(L^{\infty}(G, \mu)\right.$ is identified with the topological dual of $\left.L^{1}\right)$. The proof proceeds in some steps.

Step I. The sequence $\left(g_{n}\right)$ is relatively $L^{1}$-weak sequentially compact.

We apply Theorem 2.6. To this purpose note that $\left(g_{n}\right)$ is bounded in $L^{1}$ and moreover, for any decreasing sequence $\left(E_{k}\right) \subset \mathcal{B}(G)$, with empty intersection, one has

$$
\sup _{n \geq 0}\left|\int_{E_{k}} g_{n}(y) \mu(d y)\right| \leq 2\|f\|_{\infty} \mu\left(E_{k}\right),
$$

which tends to 0 as $k \rightarrow \infty$. Hence, possibly passing to a subsequence, still denoted by $\left(g_{n}\right)$, we know that there exists $g_{0} \in L^{1}$ such that, for any $h \in L^{\infty}(G, \mu)$,

$$
\int_{G} g_{n}(y) h(y) \mu(d y) \rightarrow \int_{G} g_{0}(y) h(y) \mu(d y), \text { as } n \rightarrow \infty .
$$

Step II. The limit function $g_{0}=2 c, \mu$-a.s.

Note that, for $x \in G$,

$$
g_{n}(x)=\int_{G} g_{n}(x-y) \tilde{\mu}(d y)=\int_{G} g_{n}(x+y) \mu(d y), \quad n \in \mathbb{N} .
$$

Set $x=0$ in (2.4). Using the $L^{1}$-weak convergence, we get

$$
2 c=\lim _{n \rightarrow \infty} g_{n}(0)=\int_{G} g_{0}(y) \mu(d y) .
$$

Now we prove that $g_{0}(x) \leq 2 c, \mu$-a.s. If this does not hold, then there exists $\epsilon>0$ such that $B=\left\{x \in G: g_{0}(x) \geq 2 c+\epsilon\right\}$ verifies $\mu(B)>0$. But then, using that $g_{n}(x) \leq 2 c, x \in G$, we find

$$
2 c \mu(B) \geq \int_{B} g_{n}(y) \mu(d y)=\int_{G} g_{n}(y) I_{B}(y) \mu(d y), \quad n \in \mathbb{N} .
$$

Passing to the limit as $n \rightarrow \infty$, we infer a contradiction. By (2.5), we get the claim.

Step III. There exists a subsequence of $\left(g_{n}\right)$, still denoted by $\left(g_{n}\right)$, which converges pointwise to $2 c, \mu$-a.s.

It is enough to show that $\left(g_{n}\right)$ converges to $2 c$ in probability (with respect to $\mu$ ). To this purpose, we write, using that $g_{n} \leq 2 c$, for any $n \geq 1$,

$$
\mu\left(x \in G:\left|g_{n}(x)-2 c\right|>\epsilon\right) \leq \frac{1}{\epsilon} \int_{G}\left(2 c-g_{n}(y)\right) \mu(d y) \rightarrow 0, \text { as } n \rightarrow \infty .
$$

Step IV. For any $x \in M_{\mu}$,

$$
\lim _{n \rightarrow \infty} g_{n}(x)=2 c
$$


By (2.4) we have, for any $x \in M_{\mu}$,

$$
g_{n}(x)=\int_{G} g_{n}(y)\left(T_{x} \circ \mu\right)(d y)=\int_{G} g_{n}(y) F^{x}(y) \mu(d y), \quad x \in M_{\mu}, n \in \mathbb{N},
$$

where $T_{x}$ is the translation operator and $F^{x}$ denotes the density of $\left(T_{x} \circ \mu\right)$ with respect to $\mu$. Now we write, for any $M>0$,

$$
\begin{aligned}
& \left|g_{n}(x)-2 c\right|=\left|\int_{G}\left(g_{n}(y)-2 c\right)\left(T_{x} \circ \mu\right)(d y)\right| \\
& \leq\left|\int_{\left\{y:\left|F^{x}(y)\right|>M\right\}}\left(g_{n}(y)-2 c\right)\left(T_{x} \circ \mu\right)(d y)\right| \\
& \quad+\left|\int_{\left\{y:\left|F^{x}(y)\right| \leq M\right\}}\left(g_{n}(y)-2 c\right) F^{x}(y) \mu(d y)\right| \\
& \leq 2\left(\|f\|_{\infty}+|c|\right)\left(T_{x} \circ \mu\right)\left(\left\{\left|F^{x}(y)\right|>M\right\}\right) \\
& \quad+\left|\int_{G}\left(g_{n}(y)-2 c\right) h^{x}(y) \mu(d y)\right|,
\end{aligned}
$$

where $h^{x}(y)=F^{x}(y) I_{\left\{y:\left|F^{x}(y)\right| \leq M\right\}}(y)$. For any $\epsilon>0$, we can choose $M>0$ and $n_{0} \in \mathbb{N}$ large enough, such that $n \geq n_{0}$ implies $\left|g_{n}(x)-2 c\right| \leq 2 \epsilon$. The claim is proved.

Final Step. Recall that $M_{\mu}$ is a semigroup in $G$; see [12, page 450]. This fact and (2.6) imply that

$$
g_{0}(k a)=2 c, \quad k \in \mathbb{N} .
$$

Now we complete the proof similarly to Choquet-Deny [3]. For any integer $m$, there exists $\hat{n}$ such that

$$
g_{\hat{n}}(k a)=\tilde{f}\left(x_{\hat{n}}+k a\right)-\tilde{f}\left(x_{\hat{n}}+(k-1) a\right)>c,
$$

for $k=1, \ldots, m$. Summing (2.8) $m$-times, we get $\tilde{f}\left(x_{\hat{n}}+m a\right)-\tilde{f}\left(x_{\hat{n}}\right)>m c$.

Letting $m \rightarrow \infty$, we find that $c \leq 0$, since $\tilde{f}$ is bounded. This means that $g(x) \leq 0, x \in G$, i.e.

$$
\tilde{f}(x) \leq \tilde{f}(x+a), \quad x \in G .
$$

Repeating the previous argument with $-f$ instead of $f$, one has $f(x)=f(x+a)$, $x \in G$. Thus (2.3) holds, for any $a \in M_{\mu}$. Now (1.1) implies that, for any $n \in \mathbb{N}$, $f * \mu^{n}(x)=f(x), \quad x \in G$. Hence (2.3) holds, for any $a \in E_{\mu}$. The assertion follows, remarking that the set of all periods of a given real function on $G$ is a subgroup of $G$. The proof is complete.

Remark 2.7. If $\mu$ is symmetric, then $\operatorname{Gr}\left(E_{\mu}\right)=E_{\mu}$, and so the formulation of Theorem 2.5 simplifies. Indeed if $\mu$ is symmetric, then $M_{\mu}$ is a subgroup of $G$. By Proposition 2.1 we know that $E_{\mu}$ is an increasing union of subgroups. Hence $E_{\mu}$ is a group.

Remark 2.8. Theorem 2.5 does not hold if we replace $E_{\mu}$ with the subgroup generated by the support $S_{\mu}$ of $\mu$.

Let $G=\mathbb{R}^{d}$ and take $\mathbb{Q}^{d}=\left\{q_{n}\right\}$ to be the set of all points in $\mathbb{R}^{d}$ having rational coordinates. Let $\left(p_{n}\right) \subset \mathbb{R}_{+}$be such that $\sum_{n \geq 1} p_{n}=1$. Define $\mu=\sum_{n \geq 1} p_{n} \delta_{q_{n}}$, where $\delta_{q_{n}}$ are Dirac measures concentrated in $q_{n}$.

Take $f=1_{\mathbb{Q}^{d}}$ to be the indicator function of $\mathbb{Q}^{d}$. It is straightforward to check that $\mu * f(x)=f(x), \quad x \in \mathbb{R}^{n}$. Moreover the support $S_{\mu}=\mathbb{R}^{d}$ and $M_{\mu}=\mathbb{Q}^{d}$. 
Note that if $h \notin \mathbb{Q}^{d}$, then $f(x+h)=f(x)$ only if $x \notin A_{h}$, where $A_{h}=-h+\mathbb{Q}^{d}$ has Lebesgue measure 0.

If we restrict our attention to bounded $\mu$-harmonic functions which are also uniformly continuous on $G$, then we can prove periodicity with respect to $\operatorname{Gr}\left(S_{\mu}\right)$. In the terminology of [4, page 396] the next result shows that any Polish abelian group has the Liouville property. We denote by $U C_{b}(G)$ the space of all uniformly continuous and bounded functions from $G$ into $\mathbb{R}$.

Theorem 2.9. Let $G$ be a Polish abelian group. Let $\mu$ be a probability measure on $G$. Let $f \in U C_{b}(G)$ be a solution to $f * \mu=f$. Then,

$$
f(x+a)=f(x), \text { for any } x \in G, a \in G r\left(S_{\mu}\right) .
$$

In [19] we provide the complete proof. It uses the same arguments given in [3], but the next lemma is needed.

Lemma 2.10. Let $G$ be an abelian Polish group. There exists a subgroup $S_{0} \subset G$, which is a countable union of compact sets and has the property that $\mu\left(S_{0}\right)=1$.

Proof. First choose compact sets $G_{n}$ such that $0 \in G_{n}, G_{n} \subset G_{n+1}$ and $\mu\left(G \backslash G_{n}\right)<$ $1 / n$.

Define new compacts $F_{n}=\left(-G_{n}\right) \cup G_{n}, n \geq 1$, and finally set

$$
K_{1}=F_{1}, K_{2}=F_{2}+F_{2}, \ldots, K_{n}=F_{n}+\ldots+F_{n} \text { (n-times), } \ldots
$$

It is easy to check that $S_{0}=\bigcup_{n \geq 1} K_{n}$ has all the required properties.

Remark 2.11. Theorem 2.5 holds more generally, with the same proof, if $G$ is a measurable abelian group; see [23, page 63]. A measurable space $(G, \mathcal{A})$ which is also a group (with additive notation) is said to be a measurable group if the group operations $(x, y) \mapsto x+y$ and $x \mapsto-x$ are both measurable (on $G \times G$ one considers the product $\sigma$-algebra $\mathcal{A} \times \mathcal{A}$ ). In measurable groups the convolution of finite measures on $\mathcal{A}$ is naturally defined. Note that a separable metric group and a locally compact group, with $\mathcal{A}$ being the Borel $\sigma$-algebra, are both examples of measurable groups.

2.3. Connections with the Choquet-Deny theorem. It is interesting to compare our result with the remarkable theorem due to Choquet and Deny, mentioned in the Introduction, valid in locally compact groups $G$; see [3]. Their theorem is concerned with the equation

$$
\nu * \mu=\nu
$$

where $\mu$ is a given probability measure on $G$ and unknown $\nu$ is a $\sigma$-finite Borel measure on $\mathrm{G}$ such that, for any compact set $K \subset G$, the Borel non-negative function

$$
G \rightarrow \mathbb{R}_{+}, \quad x \mapsto \nu(x-K)=1_{K} * \nu(x) \text { is finite and bounded on } G .
$$

It turns out that $\nu$ is periodic with periods in the subgroup generated by the support $S_{\mu}$ of $\mu$, i.e.

$$
\nu(A+h)=\nu(A), \quad h \in G r\left(S_{\mu}\right), A \in \mathcal{B}(G) .
$$

This result can be applied to the study of equation (1.1). Let us interpret function $f$ as a density of a measure $\nu$ with respect to the Haar measure $\mathcal{L}$ of $G: f=\frac{d \nu}{d \mathcal{L}}$. 
Then (2.12) implies that, for any $h \in S_{\mu}, f(x+h)=f(x), \quad \mathcal{L}$-a.s., where the set of $\mathcal{L}$-measure 0 depends, in general, on $h$; see Remark 2.8 ,

The following corollary of Theorem 2.5 can be regarded as a version of 3 , Theorem 1] in the non-locally compact case.

Proposition 2.12. Let $\mu$ be a given probability measure on a Polish abelian group $G$. Let $\nu$ be a $\sigma$-finite Borel measure on $G$ satisfying the condition (2.11) for any compact set $K \subset G$. If $\nu * \mu=\nu$, then $\nu$ is periodic with periods in $E_{\mu}$; see (2.12).

Proof. First take a compact set $K \subset G$ and consider the indicator function of $K$, i.e. $1_{K}$. We have $\left(1_{K} * \nu\right) * \mu=1_{K} * \nu$. Indeed, by the Fubini theorem,

$$
1_{K} *(\nu * \mu)(x)=\int_{G^{2}} 1_{K}(x-y-z) \nu(d y) \mu(d z)=\left(1_{K} * \nu\right) * \mu(x), \quad x \in G .
$$

By Theorem 2.5 we get that $1_{K} * \nu(x+h)=1_{K} * \nu(x), x \in G, h \in E_{\mu}$. Hence, by taking $x=0$, we get $\nu(h-K)=\nu(K)$, and so

$$
\nu(h+K)=\nu(K), \text { for any compact set } K \subset G, h \in E_{\mu} .
$$

By the inner regularity of $\nu$, for any Borel set $A \subset G$ with $\nu(A)<\infty$, there exists an increasing sequence of compact sets $\left(K_{n}\right)$, such that $K_{n} \subset A, n \in \mathbb{N}$, and $\lim _{n \rightarrow \infty} \nu\left(K_{n}\right)=\nu(A)$. It follows that $\nu(h+A)=\nu(A)$, for any $h \in E_{\mu}$. The proof is complete.

We do not know if the previous result holds when $G$ is a Polish abelian group and $E_{\mu}$ is replaced by the subgroup generated by the support of $\mu$.

Remark 2.13. Proposition 2.12 holds more generally, with the same proof, if $G$ is a Hausdorff topological abelian group. In this case we assume that the measures $\mu$ and $\nu$ are both Radon measures (a non-negative Borel $\sigma$-finite measure $\gamma$ on a Hausdorff topological space $X$ is called Radon, if for each Borel set $B \subset X$ with $\gamma(B)<\infty$, for any $\epsilon>0$ there exists a compact set $K \subset B$ such that $\gamma(B \backslash K)<\epsilon$; see for instance [23]).

\section{Convolution equations on Hilbert spaces}

3.1. The case of stable measures. Let $G=H$ be a real separable Hilbert space, $Q$ a non-negative trace class operator on $H$ and $\alpha \in(0,2]$. A probability measure $\mu$ on $H$ is said to be $(\alpha, Q)$-stable, $\alpha \in(0,2]$, centered at $x \in H$, if its characteristic function is

$$
\hat{\mu}(h):=\int_{H} e^{i\langle h, y\rangle} \mu(d y)=\exp (i\langle x, h\rangle) \exp \left(-\left(\frac{\langle Q h, h\rangle}{2}\right)^{\alpha / 2}\right), \quad h \in H ;
$$

see [16], [23], 22]. Such measures will be denoted by $N_{\alpha}(x, Q)$. Measures $N_{2}(x, Q)$ are Gaussian. In this case we also write $N(x, Q)$.

The following result extends Theorem 4.3.4 in [7].

Proposition 3.1. Let $\mu=N_{\alpha}(x, Q), \alpha \in(0,2]$. If $f \in B_{b}(H)$ solves equation (1.1), then

$$
f\left(y+Q^{1 / 2} a\right)=f(y), \quad y \in H, \quad a \in H .
$$

If, in addition, $f$ is continuous and $Q$ positive definite, then $f$ is constant on $H$.

Proof. First we show the result for $\alpha=2$. 
Lemma 3.2. Let $\mu=N(x, Q)$ and $\nu=N(y, S)$ be Gaussian measures on $H$. Then,

$$
M_{\mu * \nu}=M_{\mu}+M_{\nu}=Q^{1 / 2} H+S^{1 / 2} H .
$$

In particular $E_{\mu}=M_{\mu}=Q^{1 / 2} H$.

Proof. It is well known that $M_{\mu}=Q^{1 / 2} H$; see [7. Moreover $\mu * \nu=N(x+y, Q+S)$.

Define the linear operator $T: H \times H \rightarrow H, T(x, y)=Q^{1 / 2} x+S^{1 / 2} y$ (where as usual $\left.\left\langle(x, y),\left(x^{\prime}, y^{\prime}\right)\right\rangle:=\left\langle x, x^{\prime}\right\rangle+\left\langle y, y^{\prime}\right\rangle\right)$. We easily check that

$$
\left|(Q+S)^{1 / 2} h\right|^{2}=\left|T^{*} h\right|^{2}, \quad h \in H,
$$

where $T^{*}$ denotes the adjoint of $T$. By a classical duality argument, we have that $(Q+S)^{1 / 2} H=Q^{1 / 2} H+S^{1 / 2} H$. The proof is complete.

Continuing the proof of the proposition note that by Lemma 3.2 $Q^{1 / 2} H=E_{N_{2}}$. Thus Theorem 2.5 gives the first claim. The second one follows from the density of $Q^{1 / 2} H$ in $H$ when $Q$ is non-degenerate.

Let us now consider $\alpha \in(0,2)$ and set $N_{\alpha}=N_{\alpha}(x, Q)$. We show that $Q^{1 / 2} H \subset$ $M_{N_{\alpha}}, \alpha \in(0,2]$. For this we use subordination. Let $\nu_{\alpha}$ be an $\alpha$-stable distribution $\nu^{\alpha}$ on $[0,+\infty)$, with the Laplace transform given by

$$
\int_{0}^{\infty} e^{-\lambda s} \nu^{\alpha}(d s)=e^{-(\lambda)^{\alpha / 2}}, \lambda>0
$$

It is easy to check that

$$
N_{\alpha}(B):=\int_{0}^{\infty} N_{2}(x, s Q)(B) \nu^{\alpha}(d s), \quad B \in \mathcal{B}(H) .
$$

Take $A \in \mathcal{B}(H)$ such that $N_{\alpha}(A)=0$; then, $N_{2}(x, s Q)(A)=0$, for any $s \in S_{\nu^{\alpha}}$ $=\mathbb{R}_{+}$. Let $g=Q^{1 / 2} h$, for some $h \in H$. By the absolute continuity of the Gaussian measures,

$$
\left(T_{g} \circ N_{\alpha}\right)(A)=N_{\alpha}(A+g)=\int_{0}^{\infty} N_{2}(x-g, s Q)(A) \nu^{\alpha}(d s)=0 .
$$

Hence, $Q^{1 / 2}(H) \subset M_{N_{\alpha}}$. By Theorem 2.5 we get the claim.

For information about the set of all admissible shifts for general $\alpha$-stable measures, we refer to [2] and [24].

3.2. Liouville type theorems on Hilbert spaces. Let $\mu_{t}, t \geq 0$, be a convolution semigroup of measures on a real separable Hilbert space $H$. This means that $\mu_{t} * \mu_{s}=\mu_{t+s}, t, s \geq 0, \mu_{0}$ is the Dirac measure concentrated in 0 and $\mu_{t}$ is weakly continuous at $t=0$. Let $P_{t}$ be the Markovian convolution semigroup determined by $\mu_{t}, t \geq 0$,

$$
P_{t} f(x)=\int_{H} f(x-y) \mu_{t}(d y)=f * \mu_{t}(x), \quad x \in H, t \geq 0, f \in B_{b}(H) .
$$

See [16], 23], [13] and 22] for more information on convolution semigroups and Lévy processes. A function $h \in B_{b}(H)$ is said to be a bounded harmonic function for $P_{t}$, briefly a BHF for $P_{t}$ (see [1], [9], 17] and [18]), if

$$
P_{t} h=h, \quad t \geq 0 \text {. }
$$


In particular, when $P_{t}$ is a compound convolution semigroup, i.e. $P_{t} f(x)=e^{-\lambda t}$ $\sum_{k \geq 0} \frac{(t \lambda)^{k}}{k !}\left(f *(\nu)^{k}\right)(x)$, where $\lambda>0$ and $\nu$ is a given probability measure on $H$, one has that $h$ is a BHF for $P_{t}$ if and only if $h$ is a bounded $\nu$-harmonic function.

We now present a Liouville type theorem about BHFs for convolution semigroups. The first part is a consequence of Theorem 2.5. The second one is a generalization of a surprising result obtained by Goodman 14. It states that in infinite dimensions there exist non-constant BHFs for the heat semigroup (see also [7], Section 4.3.1).

Theorem 3.3. 1) Let $P_{t}$ be the Markovian semigroup (3.6) on a separable Hilbert space $H$ and let

$$
\Gamma=\operatorname{Gr}\left(\bigcup_{t \geq 0} M_{\mu_{t}}\right)
$$

Then each BHF $h$ for $P_{t}$ is periodic with periods in $\Gamma$. If $h$ is continuous and $\bar{\Gamma}=H$, then $h$ is constant.

2) For arbitrary semigroups (3.6) there exists a non-constant BHF $\phi$ if $H$ is infinite dimensional.

Proof. 1) It follows from Theorem 2.5. We only note that, by Proposition 2.1, one has $\operatorname{Gr}\left(\bigcup_{t \geq 0} E_{\mu_{t}}\right)=\Gamma$.

2) We use a probabilistic representation of convolution semigroups; see 13 .

There exists a Lévy process $\left(Z_{t}\right)$ on a stochastic basis $\left(\Omega, \mathcal{F},\left(\mathcal{F}_{t}\right)_{t \geq 0}, \mathbb{P}\right)$, with values in $H$, such that the law of each $Z_{t}$ is $\mu_{t}, t \geq 0$. The process $\left(Z_{t}\right)$ can be represented as

$$
Z_{t}=a t+\eta_{t}+\xi_{t}, \quad t \geq 0
$$

where $a \in \mathbb{R}^{n},\left(\eta_{t}\right)$ is a square integrable martingale and $\left(\xi_{t}\right)$ is a compound Poisson process. Moreover the processes $\left(\eta_{t}\right)$ and $\left(\xi_{t}\right)$ are independent, and so in particular

$$
P_{t} f(x)=\mathbb{E} f\left(x-\xi_{t}-\eta_{t}-a t\right)=f * \nu_{t} * r_{t}(x),
$$

where $\nu_{t}$ is the law of $a t+\eta_{t}$ and $r_{t}$ the law of $\xi_{t}$. Thus it is enough to construct a non-constant function $\phi$ such that

$$
\phi * \nu_{t}=\phi \text { and } \phi * r_{t}=\phi, \quad t \geq 0
$$

Let us first consider $\eta_{t}+$ at with law $\nu_{t}$. Remark that there exists a non-negative trace class operator $Q: H \rightarrow H$, such that the following holds:

$$
\langle Q h, k\rangle=\frac{1}{t} \mathbb{E}\left(\left\langle\eta_{t}, h\right\rangle\left\langle\eta_{t}, k\right\rangle\right), \quad t>0, h, k \in H .
$$

Let us choose an orthonormal basis $\left(e_{k}\right)$ in $H$, such that $Q e_{k}=\lambda_{k} e_{k}$. We have that $\sum_{k \geq 1} \lambda_{k}<\infty$. Let $\left(\alpha_{k}\right)$ be a sequence of positive numbers, diverging to $+\infty$, such that

$$
\sum_{k \geq 1} \lambda_{k} \alpha_{k}+\sum_{k \geq 1} a_{k}^{2} \alpha_{k}<\infty, \quad a_{k}=\left\langle a, e_{k}\right\rangle, \quad k \in \mathbb{N}
$$

and define the linear subspace $K$,

$$
K=\{x \in H: g(x)<\infty\}, \quad g(x)=\sum_{k \geq 1} x_{k}^{2} \alpha_{k}, \quad x \in H, x_{k}=\left\langle x, e_{k}\right\rangle .
$$


Since $\left(\alpha_{k}\right)$ diverges, one has that $K$ is strictly contained in $H$. Moreover $a \in K$ by construction. It turns out that the law of $\eta_{t}+a t$ is concentrated on $K$, for any $t \geq 0$. Indeed one has

$$
\mathbb{E} g\left(\eta_{t}+a t\right)=\sum_{k \geq 1} \alpha_{k}\left\langle\eta_{t}, e_{k}\right\rangle^{2}+t \sum_{k \geq 1} a_{k}^{2} \alpha_{k}<\infty
$$

and so $\eta_{t}+a t$ is almost surely in $K$, for any $t \geq 0$. Note that

$$
\left(I_{K}\right) * \nu_{t}(x)=\int_{H} I_{K}(x-y) \nu_{t}(d y)=\nu_{t}(x-K)=I_{K}(x), x \in H, t \geq 0 .
$$

Indeed if $x \notin K$, then $x-k \notin K$, for any $k \in K$. This gives that $I_{K}$ is a non-constant BHF for the convolution semigroup determined by the process $\left(\eta_{t}+a t\right)$.

Let us consider the remainder compound Poisson process $\left(\xi_{t}\right)$; see (3.9). Denote by $\lambda>0$ its intensity, by $\nu$ its Lévy measure and by $S_{t}$ the associated convolution semigroup. One has

$$
S_{t} f(x)=\mathbb{E} f\left(x-\xi_{t}\right)=e^{-\lambda t} \sum_{k \geq 0} \frac{(t \lambda)^{k}}{k !}\left(f *(\nu)^{k}\right)(x),
$$

where $\mathbb{E}\left(e^{i\left\langle\xi_{t}, h\right\rangle}\right)=e^{-t \psi(h)}, h \in H, t \geq 0$, and $\psi(h)=\int_{H}\left(e^{i\langle x, h\rangle}-1\right) \nu(d x)$. As already noted, $h \in B_{b}(H)$ is a BHF for $S_{t}$ if and only if $h * \nu=h$.

Let us first construct a non-constant bounded $\nu$-harmonic function $h$. Let us introduce $\lambda_{k}^{\prime}=\mathbb{E}\left(e^{-|U|}\left\langle U, e_{k}\right\rangle^{2}\right)$, where $U: \Omega \rightarrow H$ is a random variable with law $\nu$. It is clear that

$$
\sum_{k \geq 1} \lambda_{k}^{\prime}=\mathbb{E}\left(e^{-|U|}|U|^{2}\right)<\infty
$$

Take a diverging sequence of positive real numbers $\left(\alpha_{k}^{\prime}\right)$, such that $\sum_{k \geq 1} \lambda_{k}^{\prime} \alpha_{k}^{\prime}<\infty$ and define the linear subspace $K^{\prime}, K^{\prime}=\left\{x \in H: \sum_{k \geq 1} x_{k}^{2} \alpha_{k}^{\prime}<\infty\right\}$. One has that $h$ is a non-costant BHF for $S_{t}$.

To finish the proof, define $\tilde{\alpha}_{k}=\min \left(\alpha_{k}, \alpha_{k}^{\prime}\right)$, and introduce the new subspace $\tilde{K}=\left\{x \in H, \sum_{k \geq 1} x_{k}^{2} \tilde{\alpha}_{k}<\infty\right\}$. Repeating the previous arguments, we find that $\phi=I_{\tilde{K}}$ is non-constant and verifies (3.11). This completes the proof.

We recall that a Markovian semigroup $P_{t}$, acting on $B_{b}(H)$, is called strong Feller if $P_{t} f$ is continuous on $H$, for any $f \in B_{b}(H), t>0$; see for instance [7].

Corollary 3.4. Markovian convolution semigroups $P_{t}$, given by (3.6) on an infinite dimensional Hilbert space $H$, are never strong Feller.

\section{ACKNOWLEDGMENTS}

The authors thank the Institute of Mathematics of the Polish Academy of Sciences in Warsaw and Scuola Normale Superiore di Pisa, where parts of this paper were prepared, for good working conditions.

\section{REFERENCES}

[1] R. M. Blumenthal and R. K. Getoor, Markov processes and potential theory, Pure and Applied Mathematics, Academic Press (1968) New York-London. MR0264757 (41:9348)

[2] S. Cambanis and M. Marques, Admissible and singular translates of stable processes, Probability theory on vector spaces IV, Lecture Notes in Math. 1391 (1989) 239-257. MR.1020566 (91h:60044) 
[3] G. Choquet and J. Deny, Sur l'equation de convolution $\mu=\mu * \sigma$, C. R. Acad. Sci. Paris 250 (1960) 799-801. MR0119041 (22:9808)

[4] C.-H. Chu and C.-W. Leung, The convolution equation of Choquet and Deny on [IN]-groups, Integral Equations Operator Theory 40 (2001) 391-402. MR.1839466 (2002e:43001)

[5] C.-H. Chu, T. Hilberdink, and J. Howroyd, A matrix-valued choquet deny theorem, Proc. Amer. Soc. 129 (2000) 229-235. MR1784024 (2001j:43007)

[6] C.-H. Chu and A. T.-M. Lau, Harmonic functions on groups and Fourier algebras, Lecture Notes in Mathematics 1782, Springer-Verlag (2002) Berlin. MR1914221 (2003i:43001)

[7] G. Da Prato and J. Zabczyk, Second Order Partial Differential Equations in Hilbert Spaces, London Mathematical Society Lecture Note Series 293, Cambridge University Press (2002). MR.1985790 (2004e:47058)

[8] N. Dunford, J. T. Schwartz, Linear operators. Part I. General theory, Pure and Applied Mathematics, Vol. 7, Interscience Publishers, Inc. (1958) New York. MR1009162 (90g:47001a)

[9] E. B. Dynkin, Markov Processes, Vol. I-II, Springer-Verlag (1965) Berlin-GöttingenHeidelberg. MR0193671 (33:1887)

[10] W. Feller, An introduction to probability theory and its applications, Vol. II, second edition, John Wiley \& Sons, Inc. (1971) New York-London-Sydney. MR0270403 (42:5292)

[11] S. R. Foguel, The ergodic theory of Markov processes, Van Nostrand Mathematical Studies 21 (1969) New York-Toronto. MR0261686 (41:6299)

[12] I. I. Gihman and A. V. Skorohod, The Theory of Stochastic Processes, Vol. I, Springer-Verlag (1974) Berlin. MR0346882 (49:11603)

[13] I. I. Gihman and A. V. Skorohod, The Theory of Stochastic Processes, Vol. II, SpringerVerlag (1974) Berlin. MR0375463 (51:11656)

[14] V. Goodman, A Liouville theorem for abstract Wiener spaces, Amer. J. Math. 95 (1973) 215-220. MR0322971 (48:1329)

[15] B. E. Johnson, Harmonic functions on nilpotent groups, Integr. Equ. Oper. Theory 40 (2001) 454-464. MR.1839470 (2002c:43002)

[16] K. R. Parthasarathy, Probability measures on metric spaces, Academic Press (1967) New York and London. MR0226684 (37:2271)

[17] R. G. Pinsky, Positive Harmonic Functions and Diffusion, Cambridge Studies in Advanced Mathematics 45 (1995). MR:1326606 (96m:60179)

[18] E. Priola and J. Zabczyk, Liouville Theorems for non-local operators, J. Funct. Anal. 216 (2004) 455-490. MR2095690 (2005g:35315)

[19] E. Priola and J. Zabczyk, On bounded solutions to convolution equations, Preprint 11, Department of Mathematics, University of Turin (2005), see http://www2.dm.unito.it/paginepersonali/priola/index.htm.

[20] C. R. Rao and D. N. Shanbhag, Choquet-Deny type functional equations with applications to stochastic models, John Wiley \& Sons (1994). MR.1329995 (97d:60020)

[21] A. Raugi, A general Choquet-Deny theorem for nilpotent groups, Ann. Inst. H. Poincaré Probab. Statist. 40 (2004) 677-683. MR2096214 (2005h:60016)

[22] K-I. Sato, Lévy processes and infinite divisible distributions, Cambridge University Press (1999). MR:1739520 (2003b:60064)

[23] N. N. Vakhania, V. I. Tarieladze, and S. A. Chobanyan, Probability distributions on Banach spaces, Mathematics and its Applications, D. Reidel Publishing Co. (1987) Dordrecht. MR $1435288(97 \mathrm{k}: 60007)$

[24] J. Zinn, Admissible translates of stable measures, Studia Math. 54 (1976) 245-257. MR0400376 (53:4210)

Dipartimento di Matematica, Università di Torino, via Carlo Alberto 10,10123 , TORINO, ITALY

E-mail address: priola@dm.unito.it

Instytut Matematyczny, Polskiej Akademin Nauk, ul. Sniadeckich 8, 00-950, WarSZAWA, POLAND

E-mail address: zabczyk@impan.gov.pl 